\title{
Void Traversal for Guaranteed Delivery in Geometric Routing
}

\author{
Mikhail Nesterenko and Adnan Vora \\ Computer Science Department \\ Kent State University \\ Kent, OH, 44242 \\ mikhail@cs.kent.edu,avora@cs.kent.edu
}

\begin{abstract}
Geometric routing algorithms like GFG (GPSR) are lightweight, scalable algorithms that can be used to route in resource-constrained ad hoc wireless networks. However, such algorithms run on planar graphs only. To efficiently construct a planar graph, they require a unit-disk graph. To make the topology unit-disk, the maximum link length in the network has to be selected conservatively. In practical setting this leads to the designs where the node density is rather high. Moreover, the network diameter of a planar subgraph is greater than the original graph, which leads to longer routes. To remedy this problem, we propose a void traversal algorithm that works on arbitrary geometric graphs. We describe how to use this algorithm for geometric routing with guaranteed delivery and compare its performance with GFG.
\end{abstract}

\section{INTRODUCTION}

Geometric routing is a promising approach for message transmission in ad hoc wireless networks. Unlike traditional ad hoc routing, geometric routing algorithms have no control messages or routing tables, the nodes maintain no information about data messages between transmissions and each data message is of constant size. Hence, geometric routing is quite scalable. Geometric routing is particularly appropriate for wireless sensor networks. Networked sensors usually have limited resources for routing information, yet many applications for sensor networks require ad hoc configurations of large scale.

In geometric routing, each node knows it own geographic coordinates. Each node is also aware of the coordinates of other nodes and links in a part of the network around it. The coordinates are either obtained from GPS receivers or a localization algorithm [1]. The source node of a data message has the coordinates of the target but the source does not know the complete route to it. The source selects one of its neighbors and sends the message to it. After getting the message the receiver forwards the message further. The objective of the algorithm is to deliver the message to the target.

The simplest version of a geometric routing algorithm is greedy routing. In greedy routing the source, as well as each intermediate node, examines its neighboring nodes and forwards the message to the one that is the closest to the target. Unfortunately, the greedy routing algorithm fails if the message recipient is local minimum: all its neighbors are further away from the target than the node itself. In compass routing [2], the next hop is selected such that the angle between the direction to the next hop node and to the destination is minimal. However, compass routing livelocks even on planar graphs.

GFG [3] (also known as GPSR [4]) is the first algorithm that guarantees delivery of the message. The algorithm is designed for planar graphs. It uses greedy routing. To get out of a local minimum GFG sequentially traverses the faces of the planar graph that intersect the line between the source and target. GFG assumes that the original communications graph is a unit-disk graph. For this graph, the authors construct its Gabriel subgraph. This subgraph preserves the connectivity of the original graph and it can be constructed locally by each node. Datta et al [5] propose further improvements to GFG. Kuhn et al [6] present a similar algorithm with asymptotically optimal worst-case performance.

Our contribution. The guaranteed delivery geometric routing algorithms, of which we are aware, have the following shortcoming. Their local minimum avoidance part runs over a planar graph. The efficient construction of a planar graph requires that the original graph is unit-disk. As recent work [7] demonstrates this assumption is not realistic for connection topologies formed by common networked sensors such as Berkeley motes [8]. Motes' radio propagation patters prove to be quite intricate.

In this paper we demonstrate how to carry out geometric routing with guaranteed delivery on arbitrary non-planar graphs. The foundation of our approach is a void traversal algorithm. We describe algorithms VOID-1 and VOID-2 that incorporate void traversal for routing across the whole network. We also present GVG - an algorithm that uses greedy routing and void traversal to get out of local minima. The void traversal is based on each node storing the network topology of its neighborhood. The neighborhood size needs to meet a condition we call intersection semi-closure. Most practical geometric graphs meet this condition such that the storage requirements for each node are independent of the size of the network and rather small.

We compared the performance of VOID-2 with FACE-2 which is the foundation of GFG. We used randomly generated graphs as a base for comparison. A large number of graphs did 
not have unit-disk subgraphs. Hence FACE-2 cannot be run. On graphs where FACE-2 and VOID-2 are run, depending on the graph generation parameters, the paths selected by VOID2 were $35-75 \%$ shorter. The memory storage requirements for VOID-2 were independent of the network size and only modestly increased compared to those of FACE- 2 .

Paper organization. This paper is organized as follows. We give definitions and describe our notation in Section III We present our void traversal algorithm in Section [III. In Section IV we describe how this algorithm can be used for geometric routing. In Section $\mathrm{V}$ we describe the results of the performance comparison of the void traversal versus face traversal algorithms. Section VI concludes the paper.

\section{Preliminaries}

Notation. We model a sensor network as a geometric graph. A geometric graph $G=(V, E)$ is a set of nodes (vertices) $V$ on a Euclidean plane connected by edges $E$. The number of nodes is $n=|V|$. Such graph is planar if its edges intersect only at vertices. Void is a region on a plane such that any two points of this region can be connected by a curve that does not intersect any of the edges of the graph. A boundary of a void contains the segments of the edges adjacent to the void. In every finite graph there is one unbounded external void. A void in a planar graph is face. Observe that the boundary of a face forms a simple cycle. Moreover, each edge of a planar graph borders at most two faces. An edge of a non-planar graph may contain the segments of the borders of arbitrarily many faces.

Neighborhood $N(u)$ of a node $u$ is a subgraph of $G$. A neighborhood relation $\mathcal{N}(G)$ over graph $G$ associates each vertex of $V$ with a subgraph of $G$. Denote $(u, v) \in E$ an edge between nodes $u$ and $v$. Denote path $(u, v)$ a path from $u$ to $v$. Denote also $|u, v|$ the Euclidean distance between $u$ and $v$.

\section{Intersection semi-closure.}

Definition 1: A neighborhood relation $\mathcal{N}(G)$ over a geometric graph $G$ is d-incident edge intersection semi-closed (or just $d$-intersection semi-closed) if, for every two intersecting edges $(u, v)$ and $(w, x)$, either:

- there exist path $(u, w)$, path $(u, x)$, either one no more than $d$ hops and $(w, x), \operatorname{path}(u, w)$ and $\operatorname{path}(u, x)$ belong to $N(u)$; or

- there exist $\operatorname{path}(v, w)$, path $(v, x)$, either one no more than $d$ hops and $(w, x), \operatorname{path}(v, w)$, and $\operatorname{path}(v, x)$ belong to $N(v)$.

The attractive feature of the intersection semi-closed neighborhood relation is that for any edge $(u, v)$, the information about every edge that intersects $(u, v)$ as well as how to reach such edge is contained in the union of $N(u)$ and $N(v)$. Observe that for a particular graph, depending on the value of $d$, such a relation may not exist. However, for any graph there always exists a $n$-incident edge semi-closed relation.

To illustrate Definition 1, we apply it to unit-disk graphs. Unit-disk graph is a geometric graph where two nodes $u$ and

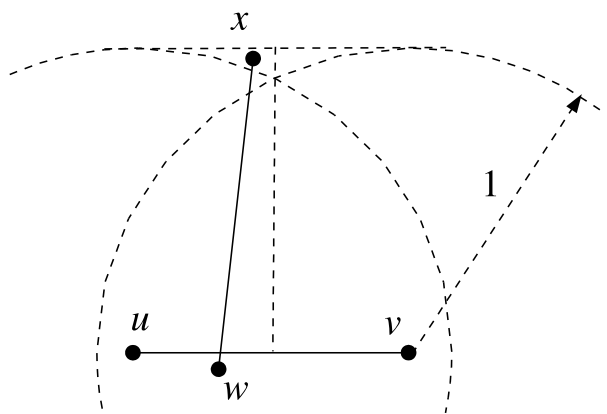

Fig. 1. Edge $(w, x)$ belongs to $N(u)$ in a unit-disk graph

$v$ are connected by an edge if an only if $|u, v| \leq 1$.

Lemma 1: A neighborhood relation over a unit-disk graph is 2-intersection semi-closed if for every node $u$ and every edge $(w, x)$ such that $|u, w| \leq 1$ and $|u, x| \leq 2 / \sqrt{3}$ it follows that $(u, w) \in N(u)$.

Proof: By definition $\mathcal{N}(G)$ is 2-intersection semi-closed if for every edge $(u, v)$ and every edge $(w, x)$ intersecting $(u, v)$, and the route to $w$ and $x$ are either in $N(u)$ or $N(v)$. These routes are no longer than 2 hops.

Let $N(u)$ and $N(v)$ be according to the conditions of the lemma. Since $G$ is unit-disk, if $|u, w| \leq 1$ and $|u, x| \leq 1$ then $(u, w) \in E$ and $(u, x) \in E$. According to the conditions of the theorem, both of these edges as well as $(w, x)$ itself belong to $N(u)$. If this is the case, then both the route to the endpoints and the edge itself belong to $N(u)$. Moreover, these routes are at most 1-hop long. Hence, the definition of intersection semi-closure is satisfied. Similar argument applies to $v$.

Suppose that $|u, x|>1$ and $|v, x|>1$. Refer to Figure 1 for illustration. Since $(w, x)$ intersects $(u, v), x$ could not be further away from $(u, v)$ than 1 . Hence, $|u, w|<1$ and $|v, w|<1$. Since $G$ is a unit-disk, $(u, w) \in G$ and $(v, w) \in G$. By the conditions of the lemma $(u, w) \in N(u)$ and $(v, w) \in N(v)$. Suppose that $x$ is closer to $u$ than to $v$. Then, the the distance between $x$ and $u$ is at most $2 / \sqrt{3}$. By the conditions of the lemma $(w, x) \in N(u)$. Which means that $N(u)$ contains the edge $(w, x)$ and, since $N(u)$ also contains $(u, w), N(u)$ contains the routes to both $w$ and $x$. Moreover, the path $(u, x)$ is just 2-hops long: $(u, w),(w, x)$. The argument for $v$ is similar. The lemma follows.

Geometric routing. Consider a connected geometric graph $G=(V, E)$, a neighborhood relation $\mathcal{N}(G)$ over it and a pair of nodes $(s, t) \in V$. Source $s$ has a message to transmit to target $t$. The source knows the coordinates of the target. The message may be transmitted via intermediate nodes. Each node may potentially add data to the transmitted message. In the sequel we ignore the payload of the message and assume that it contains only the routing information.

A routing algorithm specifies a procedure for intermediate node selection. A geometric routing algorithm with guaranteed delivery ensures the eventual message delivery under the 


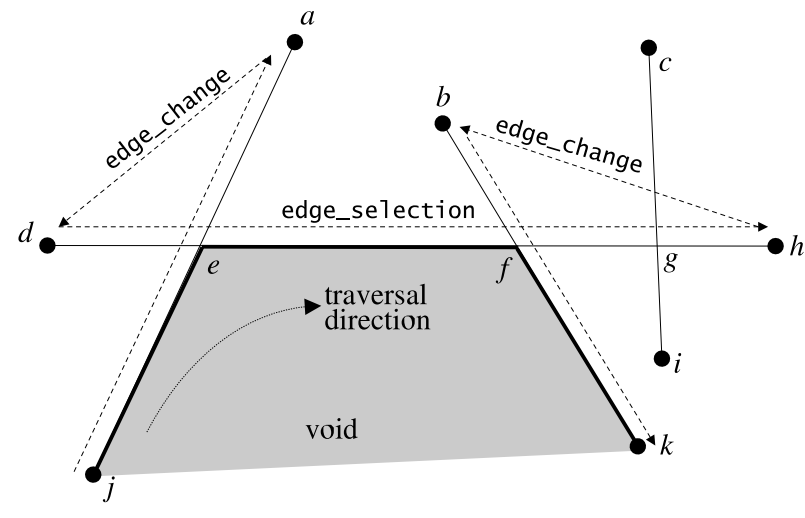

Fig. 2. Traversal of void jefk.

following two constraints: each node $u$ receiving the message, selects the next node only on the basis of $N(u)$ and the contents of the message $u$ received; the message size is independent of network size. Observe that the nodes are not allowed to keep any information about the transmitted message after it has been forwarded.

\section{VoID TRAVERSAL Algorithm}

Overview. The algorithm traverses an internal void clockwise following the segments of the edges that comprise the border of the void. The external void is traversed counter-clockwise. Refer to Figure 2 for the illustration. Given an edge, for example $(d, h)$, that contains the segment of the void's border, the algorithm has to select the next edge. For this the algorithm needs to determine the ends of the segment of $(d, h)$ that borders the void. One of the ends $-e$ is the intersection of the previous edge $(a, j)$ and $(d, h)$. The other end $-f$ is the point of the intersection of $(d, h)$ and another edge $(b, k)$ such that $f$ is closest to $e$ in the direction of the traversal of the void.

Recall that in the intersection semi-closed neighborhood relation, the union of the neighborhoods of $d$ and $h$ contains every edge intersecting $(d, h)$ as well as a route to this edge. Hence, to accomplish its objective the algorithm has to examine the neighborhoods of $d$ and $h$.

Details. The algorithm uses two types of messages: edge_change, and edge_selection. edge_change contains: previous edge, current edge and the direction of the traversal of the current edge. edge_selection message contains: previous edge, and suggested next edge.

When a node $d$ receives edge_change message from node $a$, it determines the intersection point $e$ between the previous edge $(a, j)$ and the current edge $(d, h)$. Notice that the intersection point may be $d$ itself. Then $d$ examines $N(d)$ to find the edge whose intersection point is the closest to $e$ in the direction of traversal. This edge is the suggested next edge. Note that the graph is intersection semi-closed and $N(d)$ may not contain some of some edges that intersect $(d, h)$. For example: $(b, k) \notin N(d)$.
Hence, $d$ selects $(c, i)$ as the suggested next edge. Edge $(c, i)$ intersects $(d, h)$ at point $g$. If $d$ does not find any edge that intersects $(d, h)$, it keeps the suggested next edge field empty. Node $d$ sends edge_selection to the other node $h$ incident to the current edge.

When node $h$ receives edge_selection from $d, h$ compares the suggested next edge $(c, i)$ that $h$ receives from $d$ with the edges in $N(h)$. If $h$ finds an edge $(b, k) \in N(h)$ intersecting $(d, h)$ whose intersection point $f$ is closer to $e$ than $g$, then $h$ makes $(b, k)$ the suggested next edge. Otherwise, the suggested next edge remains the same. If neither $d$ nor $h$ find the suggested next edge in this manner, $h$ considers the edges incident to itself. Node $h$ selects the edge nearest to $(d, h)$ counter-clockwise.

Node $h$ forms an edge_change message. This message contains $(d, h)$ as the previous edge and $(b, k)$ as the current edge. From the information contained in edge_selection that $h$ receives from $d, h$ is able to determine the direction of the traversal of $(d, h)$. The traversal direction is included in edge_change.

After composing edge_change, $h$ sends this message to either $b$ or $k$. Since the graph is intersection semi-closed, $N(h)$ may not contain the route to either nodes. In this case $h$ returns the message to $d$ and $d$ forwards it to the appropriate node.

The above discussion is summarized in the following theorem.

Theorem 1: The Void Traversal Algorithm correctly traverses an arbitrary void in a geometric graph with intersection semi-closed neighborhood relation.

\section{USING VOID TRAVERSAL TO GuARANTEE DELIVERY}

VOID-1 and VOID-2. The geometric routing algorithms we discuss are VOID-1 and VOID-2. They are rather straightforward extensions of algorithms FACE-1 and FACE-2 respectively. The latter two are planar graph geometric routing algorithms presented by Bose et al [3]. FACE-1 has a better worst-case performance. However FACE-2 is more efficient in practice. Hence, we describe VOID-2 in detail and mention briefly how VOID-1 is designed. Our algorithms are based on the following observation.

Proposition 1: Let $p_{1}$ and $p_{2} \neq p_{1}$ be two arbitrary points on the edges or vertices of a geometric graph. Let $V$ be the void such that $p_{1}$ lies on its border and the line segment $\left(p_{1}, p_{2}\right)$ intersects $V$. If there is a point $p_{3}$ where $\left(p_{1}, p_{2}\right)$ intersects the border of $V$ then $\left|p_{3}, p_{2}\right|<\left|p_{1}, p_{2}\right|$.

Both VOID-1 and VOID-2 sequentially traverse the voids that intersect the line $(s, t)$. Refer to Figure 3 for illustration. While traversing a void, VOID-1 and VOID-2 look for an intersection point with line $(s, t)$. Observe that there may be multiple such points. For example, void $V_{2}$ in Figure 3 has four such points. VOID-1 and VOID-2 differ in their actions when an intersection point is encountered. VOID-1 traverses the whole void, selects the intersection point that is closest to $t$, moves the message to this point and switches to the traversal of the next void. For example, VOID-1 would traverse 


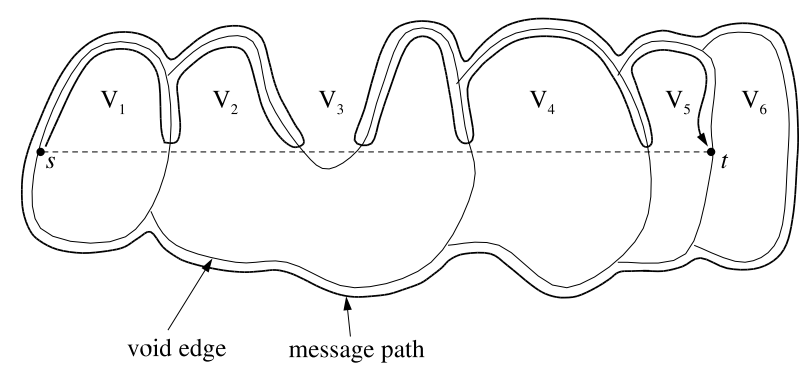

Fig. 3. Example route of VOID-2.

Algorithm: VOID-2

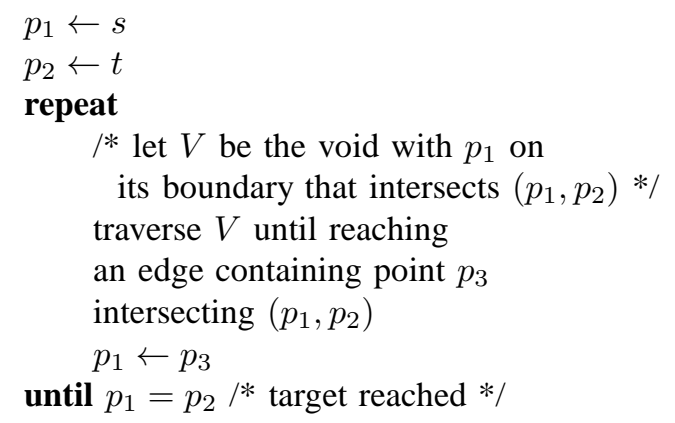

Fig. 4. Pseudocode for VOID-2. VOID-2 uses void traversal to guarantee delivery.

$V_{2}$ completely and switch to $V_{4}$. VOID-2, on the other hand, switches to the next void as soon as the first intersection point is found. The pseudocode for VOID-2 is given in Figure 4 and an example route that VOID-2 selects is shown in Figure 3

On the basis of Theorem 1 and the discussion above we state the following.

Theorem 2: Given an arbitrary geometric graph, an intersection semi-closed neighborhood relation and an arbitrary pair of nodes in this graph, both VOID-1 and VOID-2 eventually deliver a message from the first node to the second.

GVG. As in GFG, void traversal is only needed for a message to leave a local minimum. Otherwise greedy routing can be used. Care must be taken to avoid a livelock when message returns to the same local minimum. The complete algorithm greedy-void-greedy (GVG) works as follows. The message is at first forwarded according to greedy routing. When a node discovers that it is a local minimum, it notes the distance to target and the routing switches to VOID-1 or VOID-2. The routing switches back to greedy if the distance to target is less than that of the local minimum. The process repeats if necessary. Observe that, according to Theorem 2 , the delivery of a message by either VOID-1 or VOID-2 is guaranteed. Hence, either GVG eventually switches to greedy routing or delivers the message to the target. Hence the following theorem.

Theorem 3: Given an arbitrary geometric graph, an intersection semi-closed neighborhood relation and an arbitrary

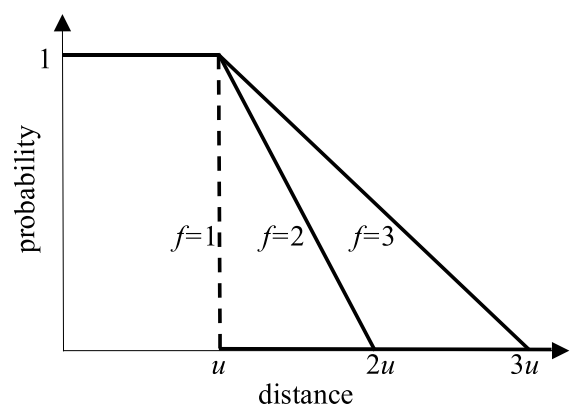

Fig. 5. Edge existence probability with respect to the distance between nodes.

pair of nodes in this graph, GVG eventually delivers a message from the first node to the second one in that pair.

\section{Performance Evaluation}

Note that the greedy phase of GFG and GVG is the same. Hence we only compare the performance of local minimum avoidance parts: void and face traversal. We use FACE-2 and VOID-2 for comparison as they are more efficient in practice then FACE-1 and VOID-1.

As a primary metric we compare the length, in the number of hops, of the routes selected by face and void traversal algorithms. Note that a void traversal algorithm makes routing decisions on the basis of larger neighborhood information. Therefore, we compared the individual node memory requirements for face and void traversal algorithms as well.

Graph Generation. We used randomly generated sets of graphs with 50, 100 and 200 nodes in a fixed area of 2 by 2 units. The nodes were uniformly distributed over the area. We used a rather simple model for fading effect of radio reception. For each set of graphs we selected the connectivity unit $u$ to be $0.3,0.25$ and 0.2 respectively. We also selected a fading factor $f$ to be either 1,2 and 3 . The connectivity between nodes was determined as follows. We deterministically added an edge for every pair of nodes that were no more than $u$ away from each other. See Figure 5. If the distance between two nodes was between $u$ and $f \cdot u$, the edge between them is added probabilistically. The probability linearly decreased from 1 to 0 . Notice that when the fading factor $f$ is equal to one, the random graphs that we generated were unit-disk.

In order to compare FACE-2 and VOID-2 we needed to compute unit-disk subgraphs of the the generated graphs. However, frequently the subgraphs were disconnected: out of 350 of randomly generated 50-node graphs with $f$ either 2 or 3 , only a single graph had a connected unit-disk subgraph. These subgraphs had to be discarded as unsuitable for FACE-2. To make the performance comparison, we adopted a different graph selection strategy. We generated random, connected unit-disk graphs and added extra links according to the connectivity rules above. Notice that this strategy favors FACE- 2 as it discounts the graphs that are not usable by FACE2. 

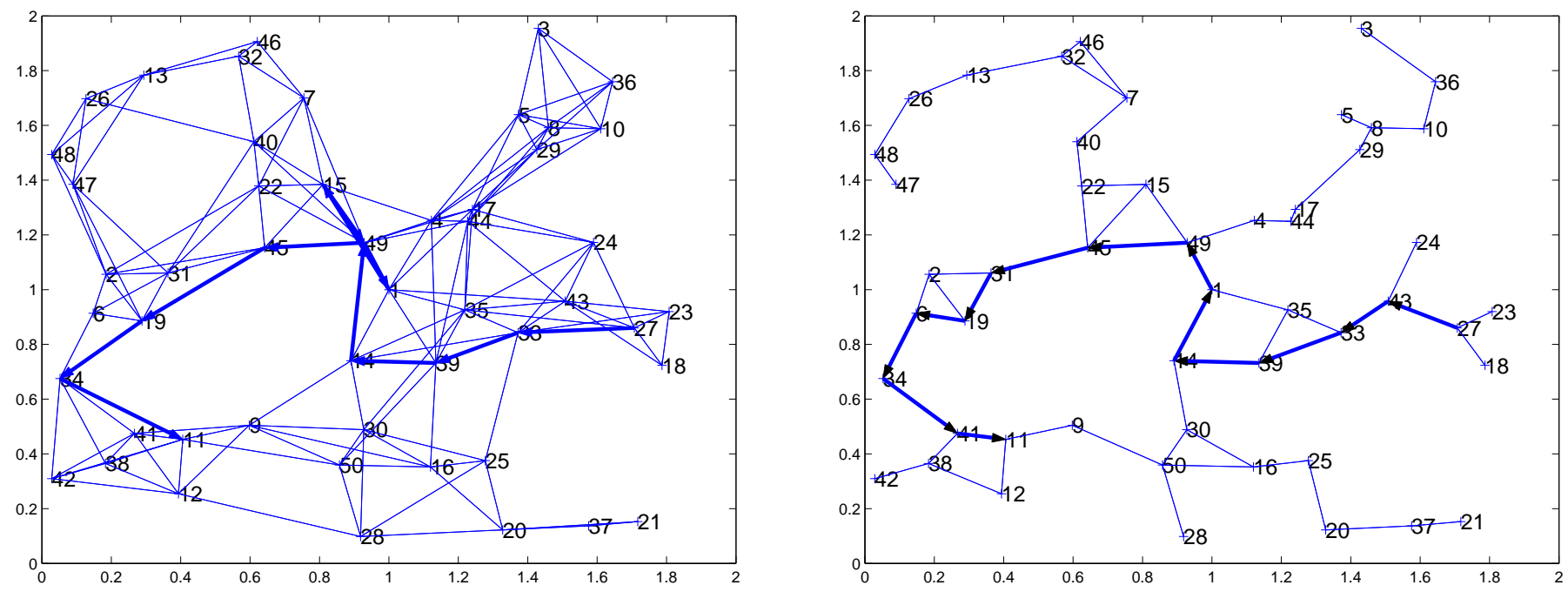

Fig. 6. Example routes selected by VOID-2 and FACE-2 between nodes 27 and 11 in a 50-node graph and its unit-disk based subgraph respectively. The fading factor $f$ is 2 .

We generated 5 graphs for each number of nodes and fading factor. This yielded the total of 45 graphs and their planar subgraphs.

Route Length Comparison. We implemented the FACE-2 and VOID-2 algorithms in Java and Matlab. We generated 10 random pairs of nodes for each set of graphs and used VOID-2 and FACE-2 to compute the routes between the nodes in each pair. VOID-2 used the original graph and FACE- 2 - its unitdisk based planar subgraph. The example routes are shown in Figure 6

We used paired comparison to estimate the improvement from FACE-2 to VOID-2 in the length of the selected routes as follows. For each pair of source and destination nodes we calculated the difference in the hopcount between routes and normalized it to the hop count of the route selected by FACE-2. That is the comparison is based on the formula: (HopCount $F A C E-$ HopCount $_{\text {VOID }}$ )/HopCount $F A C E$. Figure 7 summarizes the results. Observe that even on unit-disk graphs ( $f=$ 1), VOID-2 generates routes that are $35-45 \%$ shorter than the routes selected by FACE-2. The relative performance of VOID-2 further improves as the fading factor and density of the graphs increases.

Memory Usage Comparison. In both VOID-2 and FACE2 , each node $v$ stores the information about its neighborhood nodes $N(v)$. The size of the neighborhood differs in the two algorithms. In evaluating the memory usage, we estimated the average size of the neighborhood. For the FACE-2, the average size of the neighborhood is the average degree $d$ of the planar subgraph. In our experiments, the average size of neighborhood in VOID-2, was found to be $f \cdot d$ where $f$ is the fading factor.

\section{CONCLUSION}

The elegance and efficiency of geometric routing algorithms is remarkable. Recent advent of large-scale wireless sensor networks increased the relevance of such algorithms. However, the algorithms' demand for unit-disk based graph planarity hampered the attractiveness of geometric routing. With this paper, we lift this restriction and help the adoption of geometric routing algorithms as the routing solution of choice in sensor networks.

\section{ACKNOWLEDGMENTS}

We would like to thank Ivan Stojmenovic of the University of Ottawa and M. Kazim Khan of Kent State University for their helpful comments.

\section{REFERENCES}

[1] N. Bulusu, J. Heidemann, D. Estrin, and T. Tran, "Self-configuring localization systems: Design and experimental evaluation," ACM Transactions on Embedded Computing Systems, vol. 3, no. 1, pp. 24-60, Feb. 2004.

[2] E. Kranakis, H. Singh, and J. Urrutia, "Compass routing on geometric networks," in Proceedings of the 11th Canadian Conference on Computational Geometry (CCCG-99), Vancouver, BC, Canada, Aug. 1999, pp. $51-54$.

[3] Bose, Morin, Stojmenovic, and Urrutia, "Routing with guaranteed delivery in ad hoc wireless networks," in Wireless Networks: The Journal of Mobile Communication, Computation and Information, Kluwer, 2001, vol. 7.

[4] B. Karp and H. T. Kung, "GPSR: Greedy perimeter stateless routing for wireless networks," in Proceedings of the 6th Annual International Conference on Mobile Computing and Networking (MOBICOM-00). $\mathrm{N}$. Y.: ACM Press, Aug. 6-11 2000, pp. 243-254.

[5] S. Datta, I. Stojmenovic, and J. Wu, "Internal node and shortcut based routing with guaranteed delivery in wireless networks," Cluster Computing, vol. 5, no. 2, pp. 169-178, Apr. 2002.

[6] F. Kuhn, R. Wattenhofer, and A. Zollinger, "Asymptotically optimal geometric mobile ad-hoc routing," in Proceedings of the 6th International Workshop on Discrete Algorithms and Methods for Mobile Computing \& Communications (DialM-02). New York: ACM Press, Sept. 28 2002, pp. 24-33. 


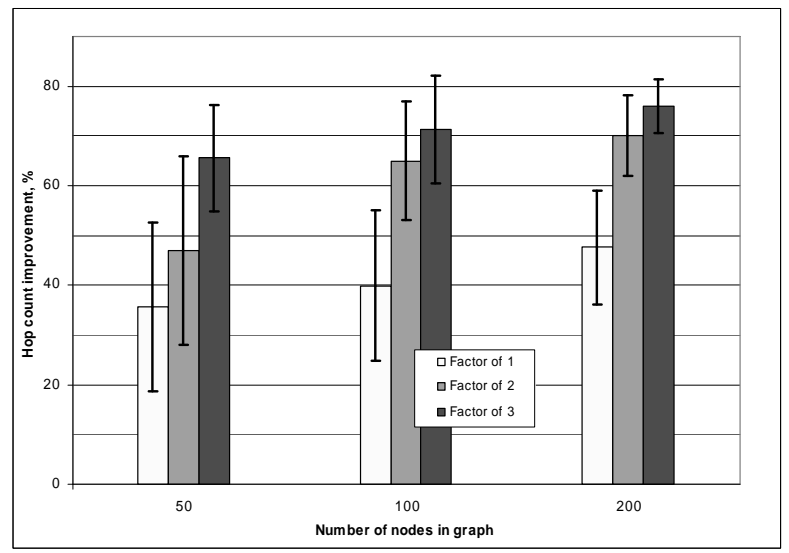

Fig. 7. Route length improvement from FACE-2 to VOID-2.

[7] D. Ganesan, B. Krishnamachari, A. Woo, D. Culler, D. Estrin, and S. Wicker, "Complex behavior at scale: An experimental study of lowpower wireless sensor networks," UCLA, Technical Report CSD-TR 020013, 2002.

[8] J. Hill and D. Culler, "Mica: A wireless platform for deeply embedded networks," IEEE Micro, vol. 22,

no. 6, pp. 12-24, Nov./Dec. 2002. [Online]. Available:

http://dlib.computer.org/mi/books/mi2002/pdf/m6012.pdf;http://www.computer.org/micro/mi2002/m6012abs.htm 